\title{
Massive mediastinal teratoma mimicking a pleural effusion on computed tomography
}

Mon Ben $\underline{\text { Chow }}^{1}$, MD, Tze Chwan $\underline{\mathrm{Lim}}^{1}$, MBBS, FRCR

ABSTRACT Mediastinal teratomas have been reported to mimic pleural effusions on chest radiography. Further evaluation of such tumours using computed tomography usually yields diagnostic characteristics that distinguish them from pleural collections. We report a patient with a mediastinal teratoma that mimicked a massive left pleural effusion on chest radiography and computed tomography.

Keywords: computed tomography, mediastinal, pleural effusion, teratoma

\section{INTRODUCTION}

Although infrequently encountered, mediastinal teratomas make up nearly $8 \%-13 \%$ of all mediastinal tumours, with peak incidence seen in young patients. ${ }^{(1)}$ Up to $75 \%$ of primary germ cell tumours of the mediastinum are mature teratomas. ${ }^{(2)}$ These tumours can be very large at presentation due to slow growth, and patients may remain asymptomatic or experience minimal symptoms. $^{(1)}$

Mediastinal teratomas are often mistaken for large pleural effusions on routine chest radiography, and computed tomography (CT) is considered the imaging modality of choice for the evaluation of these masses. ${ }^{(3)}$ We report a patient with a large, mature mediastinal teratoma that mimicked a massive pleural effusion on both chest radiography and CT imaging.

\section{CASE REPORT}

A 32-year-old Chinese woman with no past medical history presented with increasing shortness of breath over a period of four months. This was associated with productive cough. On examination, she was afebrile and was able to communicate in full sentences. Borderline tachycardia of $100 \mathrm{bpm}$ was observed and her blood pressure was 140/103 mmHg (systemic pulse pressure at $37 \mathrm{mmHg}$ ). There was tracheal deviation to the right, associated with dullness on percussion, as well as decreased breath sounds over the left chest. Routine chest radiography was performed, showing complete homogeneous opacification of the entire left hemithorax, with contralateral shift of the mediastinum (Fig. 1). Overall findings were compatible with a massive left pleural effusion. Laboratory inflammatory markers were within normal limits.

Pleural aspiration was performed without image guidance, yielding a small amount of turbid fluid. Subsequently, a large-bore chest tube was inserted, which only drained a small amount of exudative fluid. Pleural fluid cytology yielded mixed inflammatory infiltrate and reactive mesothelial cells. Bacterial

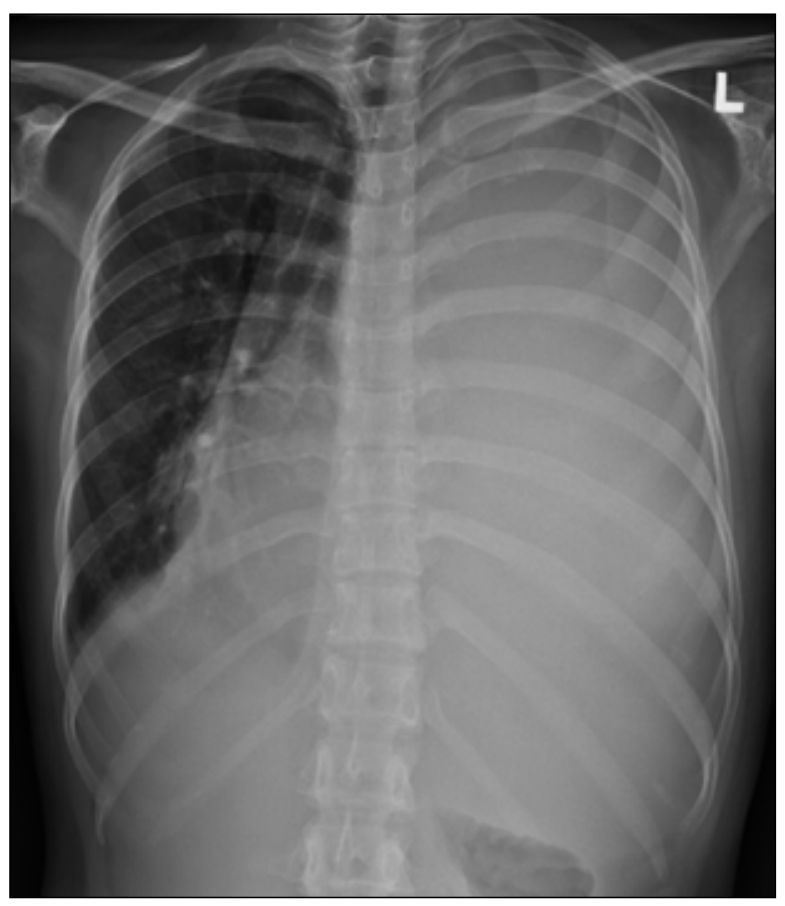

Fig. 1 Frontal chest radiograph shows homogeneous opacification of the entire left hemithorax, with shift of the mediastinal structures to the right.

and fungal cultures were negative. The patient was treated empirically with intravenous antibiotics.

A follow-up chest radiograph showed no significant interval improvement and a subsequent contrast-enhanced CT of the thorax was performed using a multi-slice scanner (SOMATOM Definition Flash; Siemens AG, Bavaria, Germany). Initial CT findings were reminiscent of a large, loculated pleural collection that may have represented an empyema. The entire left lung was collapsed, with rightward shift of the mediastinal structures, corresponding to the earlier radiographic findings (Figs. 2a \& b).

The patient remained symptomatic with persistent radiographic changes despite treatment for presumed infective pleural effusion. Laboratory inflammatory markers remained within

${ }^{1}$ Department of Diagnostic Radiology, Khoo Teck Puat Hospital, Singapore

Correspondence: Dr Chow Mon Ben, Medical Officer, Department of Diagnostic Radiology, Khoo Teck Puat Hospital, 90 Yishun Central, Singapore 768828. monben.chow@mohh.com.sg 

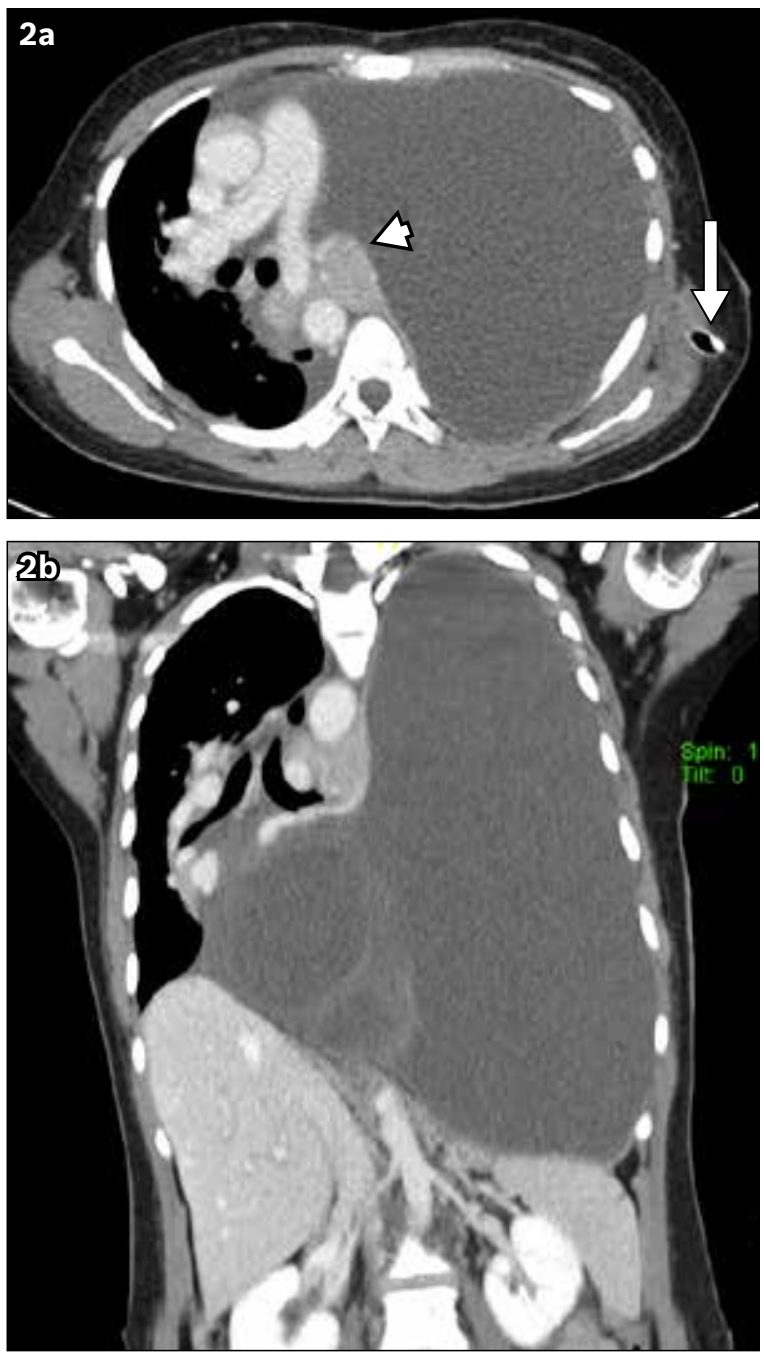

Fig. 2 (a) Axial and (b) coronal contrast-enhanced CT images show a large mediastinal teratoma. The homogenous hypo-attenuating appearance simulated a massive pleural effusion, with collapse of the left lung (arrowhead) and rightward shift of the mediastinal structures. A left chest drain was inserted (arrow)

normal limits, as were serum tumour markers, in particular alpha-foetoprotein and beta-human chorionic gonadotrophin. The patient underwent exploratory thoracotomy as definitive diagnosis could not be made.

The surgery revealed a huge mediastinal mass consisting of multiple cysts that contained serous fluid, interspersed solid components, strands of hair and specks of calcium. Histopathological examination confirmed the resected mass as a mature teratoma. The patient had uneventful postsurgery convalescence and was eventually discharged from the hospital.

\section{DISCUSSION}

It is not unusual for a mediastinal teratoma to mimic a pleural effusion on chest radiography. Many studies have reported large mediastinal teratomas, manifesting as massive pleural effusions on chest radiographs, being invariably confirmed on CT imaging. ${ }^{(1,4,5)}$ The diagnosis of a mediastinal teratoma is often clinched based on the presence of fatty or calcified components within the mass - features that are better appreciated on CT. Most teratomas tend to exhibit heterogeneous attenuation on
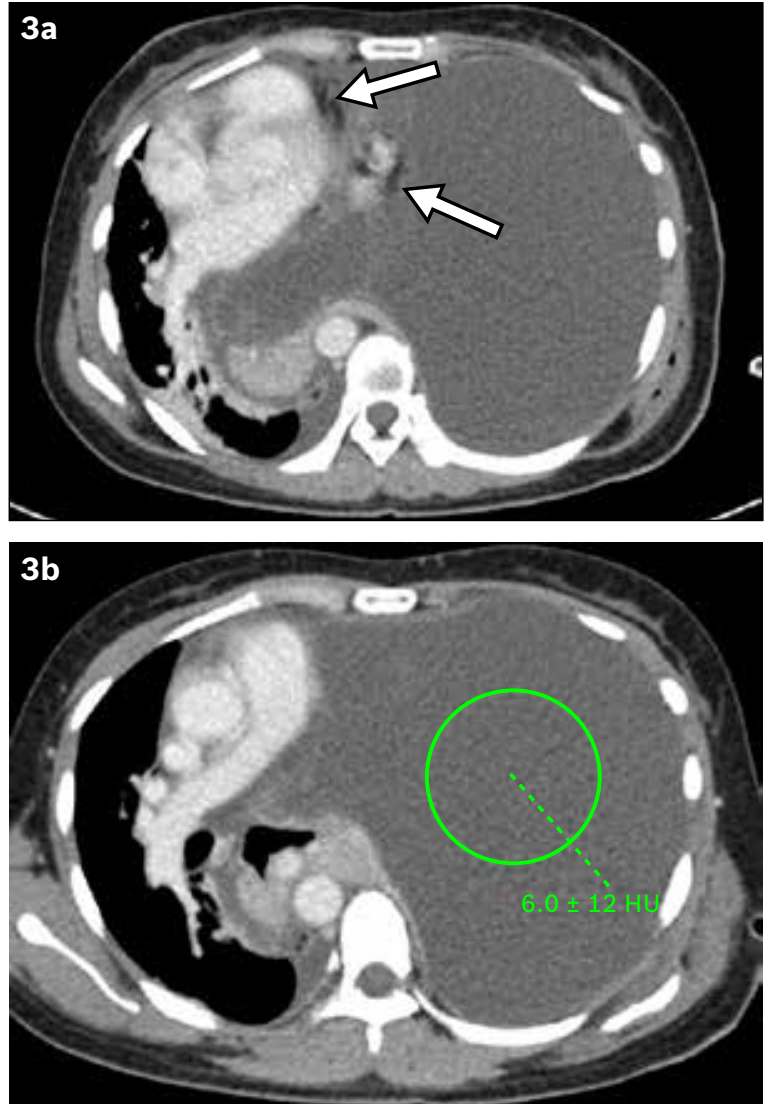

Fig. 3 Axial contrast-enhanced CT images show (a) small areas of fat attenuation (arrows) in the large mediastinal teratoma and (b) average CT attenuation (circled) of the lesion.

CT imaging. ${ }^{(3)}$ Also, some tumours may be unmistakably solid on $\mathrm{CT} .{ }^{(1,3-5)}$ Accordingly, a retrospective review of the $\mathrm{CT}$ images of our patient, following the exploratory thoracotomy, uncovered some images that showed a hint of fat attenuation within the left hemithorax (Fig. 3a). However, the average attenuation of the teratoma in our patient was about 6 Hounsfield units (Fig. 3b), further confounding our interpretation.

Although CT imaging invariably confirms mediastinal teratomas, we found that large, predominantly cystic, mediastinal teratomas have the potential to mimic large pleural effusions even on CT. We thus put forward that a large anterior mediastinal mass should be considered a possible differential diagnosis for young patients presenting with persistent or recalcitrant massive pleural effusion on chest radiography and CT.

\section{REFERENCES}

1. de Castro MA Jr, Rosemberg NP, de Castro MA, et al. Mediastinal teratoma mimicking pleural effusion on chest X-rays. J Bras Pneumol 2007; 33:113-5.

2. Nichols CR. Mediastinal germ cell tumors. Clinical features and biologic correlates. Chest 1991; 99:472-9.

3. Moeller $\mathrm{KH}$, Rosado-de-Christenson ML, Templeton PA. Mediastinal mature teratoma: imaging features. AJR Am J Roentgenol 1997; 169:985-90.

4. Sarkar A, Roy PP, Dey SK, et al. Mediastinal teratoma mimicking massive pleural effusion. J Assoc Physicians India 2010; 58:453-5.

5. Zisis C, Rontogianni D, Stratakos G, et al. Teratoma occupying the left hemithorax. World J Surg Oncol 2005; 3:76. 